(2) Open Access Full Text Article

ORIGINAL RESEARCH

\title{
Impact of serratus plane block on pain scores and incentive spirometry volumes after chest trauma
}

This article was published in the following Dove Press journal:

Local and Regional Anesthesia

\author{
Nadia Hernandez' \\ Johanna de Haan' \\ Dallis Clendeninn' \\ David E Meyer ${ }^{2}$ \\ Semhar Ghebremichael ${ }^{1}$ \\ Carlos Artime' \\ George Williams' \\ Holger Eltzschig' \\ Sudipta Sen' \\ 'Department of Anesthesiology, \\ Houston's McGovern Medical School, \\ University of Texas Health Science \\ Center, Houston, TX, 77030, USA: \\ ${ }^{2}$ Department of Surgery, Houston's \\ McGovern Medical School, University of \\ Texas Health Science Center, Houston, \\ TX, 77030, USA
}

Background: Adequate pain control is difficult to achieve in patients with multiple rib fractures (MRF). Serratus plane block (SPB) is a novel technique for alleviating rib fracture pain. Several published case reports support this hypothesis.

Purpose: The purpose of this study was to evaluate the use of SPB in MRF at our level 1 trauma center.

Methods: Our hospital's Regional Anesthesia Registry was queried for all trauma patients with MRF who underwent SPB between August 2014 and January 2018. Data were compared in each patient as a matched pair for the time periods before and after undergoing SPB. Thirty-four patients with similar baseline characteristics were enrolled.

Results: The median number of rib fractures was 7. Ordinal pain scores were found to be improved $4 \mathrm{hrs}$ after SPB from median $7 / 10$ to $3 / 10(P<0.001)$. Incentive spirometry (IS) volumes recorded 4 and 24 hrs postserratus plane block showed a median increase of 150 and $175 \mathrm{~mL}$ from baseline, respectively $(P<0.001)$. IS volumes recorded at $48 \mathrm{hrs}$ showed a median increase of $300 \mathrm{~mL}$ from baseline $(P<0.001)$. Respiratory rate decreased from a median value of 24.5 to 16 breaths $/ \min (P<0.001) . \mathrm{SpO}_{2}$ was improved at $24 \mathrm{hrs}$ from median $96 \%$ to $99 \%(P<0.001)$.

Conclusion: SPB improves pain scores and IS volumes in MRF. Because it is not limited by patient positioning or anticoagulation and has a better safety profile, it may offer a viable alternative to neuraxial techniques. Additional studies are necessary to evaluate its efficacy compared to neuraxial techniques.

Keywords: polytrauma, rib fractures, pain management, regional anesthesia

\section{Introduction}

Chest wall trauma is a significant cause of morbidity and mortality. ${ }^{1}$ In 2006 , 796,000 patients presented to emergency departments in the United States with a chest injury. ${ }^{2}$ An estimated $9.7 \%$ of trauma patients have a radiographic demonstration of rib fractures. ${ }^{1}$ Multiple rib fractures (MRF) are frequently present when the injury is caused by a high energy mechanism such as motor vehicle accidents (MVA), which include motorcycle crash, motor vehicle crash, or automobile vs pedestrian. ${ }^{3}$ Pain from MRF is associated with reduced respiratory effort, which can lead to atelectasis, inability to clear secretions, and a reduction in vital capacity. This, in turn, can result in hypoxemia, pneumonia, and acute respiratory failure. Adequate pain management of MRF not only provides symptomatic relief, it also decreases splinting and prevents secondary respiratory complications. ${ }^{4}$

Various strategies to treat chest wall pain have been utilized, including multimodal pain medication regimens, regional analgesia (intrapleural, intercostal,
Correspondence: Nadia Hernandez Department of Anesthesiology, McGovern Medical School at the University of Texas Health Science Center at Houston, 643I Fannin Street, MSB 5.030, Houston, TX 77030, USA

Tel +17137046000

Email nadia.hernandez@uth.tmc.edu 
paravertebral nerve blockade), and neuraxial analgesia (thoracic epidural analgesia (TEA), intrathecal opioids). ${ }^{5}$ Studies have shown that neuraxial analgesia is more effective than systemic opioids alone. ${ }^{6,7}$ Opioids often require high doses to adequately treat pain associated with MRF, which results in a higher incidence of narcotic-related side effects, such as sedation, respiratory depression, nausea, vomiting, and ileus. The use of neuraxial analgesia in polytrauma is frequently limited by the need for aggressive venous thromboembolic (VTE) prophylaxis with enoxaparin and subcutaneous heparin, given the exceptionally high risk of these events in this patient population. ${ }^{8}$ Furthermore, positioning of the patient for a neuraxial approach may difficult or impossible due to concomitant injuries such as unstable pelvic or spinal column fractures. Regional techniques such as intercostal nerve blocks have been studied. However, these are generally less useful due to their shorter duration and the need to perform multiple injections to cover the full dermatomal distribution. ${ }^{9}$

Ultrasound-guided serratus plane block (SPB) is a new regional technique that provides complete analgesia of the hemithorax. ${ }^{10}$ At our institution, we have had good results with this technique for patients with both penetrating and blunt chest trauma, and on our review of the literature, two case reports demonstrated the ability of SPB to provide pain control and facilitate weaning from mechanical ventilation. ${ }^{11,12}$ Therefore, we decided to incorporate the technique into analgesic regimens for MRF. The use of a serratus plane catheter has previously been described to control pain from fractured ribs 4-7 in an obese patient with multiple comorbidities, including obstructive sleep apnea. ${ }^{13}$ It has also been described in the treatment of a polytrauma patient with displaced rib fractures from ribs 2 to $9 .{ }^{14}$ We review a 3.5-year period in which 34 patients underwent ultrasound-guided SPB for MRF with the goal of improving incentive spirometry (IS) volumes and reducing pain scores.

\section{Methods}

This study was approved by the McGovern Medical School and Memorial Hermann Hospital (MHH) institutional review board (IRB). The study is a retrospective pilot study. The requirement for written informed consent was waived by the IRB, as this study was performed by retrospective chart review. The manuscript was structured to adhere to all relevant EQUATOR (STROBE) guidelines. Patient confidentiality was maintained as the data which was recorded for the study was completely deidentified. Investigators complied with the Declaration of Helsinki throughout the study process.

\section{Setting}

MHH is an American College of Surgeons-verified Level I trauma center that is the primary teaching hospital for the McGovern Medical School at the University of Texas Health Science Center at Houston.

\section{Study patients}

After approval from the University of Texas Health Science Center and MHH IRB, the regional anesthetic registry was queried for all patients that met inclusion criteria: patients aged 17 years or older who were admitted to the Trauma Service at MHH following chest wall trauma with three or more consecutive rib fractures between August 2014 and January 2018, who underwent either unilateral or bilateral ultrasound-guided SPB for pain control (see Table 1). Rib fractures were confirmed by X-ray and CT scan reads. Patients were selected for SPB at the discretion of the attending anesthesiologist managing the Acute Pain Service at the time of consult.

Exclusion criteria included: block performed following thoracic surgery, incomplete data in the electronic medical record, mechanically ventilated or sedated patients, and demented patients. Fifty patients met inclusion criteria, but 16 had one or more exclusion criteria. All patients received chemical prophylaxis against venous thromboembolism. If patients were therapeutically anticoagulated, they were not candidates for SPB catheter, but they were candidates for single- shot SPB.

For the 34 patients who were identified, their charts were retrospectively reviewed. Data analyzed included demographics, number of ribs fractured, pain score as assessed by a standard $0-10$ ordinal numeric rating scale (NRS), respiratory rate (RR), oxygen saturation $(\mathrm{SpO} 2)$, and IS volumes. Pre- and post-SPB data were collected and compared. Primary endpoints examined were: pain score, $\mathrm{RR}, \mathrm{SpO}$, and IS volumes. Total narcotic consumption was not recorded as many of these patients had other distracting injuries which may affect total opioid used. Volumes achieved on IS before

\section{Table I Patient demographics}

\begin{tabular}{|l|l|}
\hline Variable & Value \\
\hline Age, years, Median (Interquartile Range) & $57.5(49,72)$ \\
Weight, kilograms, Median (Interquartile Range) & $82(68,95)$ \\
Male sex, n (\%) & $21(62 \%)$ \\
Number of ribs fractured, Median (Interquartile & $7(6,9)$ \\
Range) & \\
Length of Hospital Stay, days, Median (Interquartile & $\mathrm{II}(7,16)$ \\
Range) & \\
\hline
\end{tabular}


and after SPB was selected as an objective measurement to assess the effectiveness of SPB in alleviating chest wall pain which would be minimally affected by distracting injuries.

\section{SPB technique}

Patients were placed in the supine position with the ipsilateral arm abducted. Anesthesiologists in our Acute Pain practice were educated on performing the SPB using the technique previously described by Blanco, et $\mathrm{al}^{10}$. The ipsilateral serratus plane was identified by ultrasound (Sonosite X-Porte, SonoSite, Bothell, WA) using a highfrequency linear transducer probe (HFL50 6-15 MHz) in a sagittal plane over lateral chest wall. Patients were scanned from the anterior to the posterior axillary line to find the optimal image and injection site, avoiding chest tubes, dressings, and subcutaneous air artifact where necessary. The serratus anterior muscle originates from ribs 1 through 9 and inserts on the medial border of the ipsilateral scapula. The optimal ultrasound image is demonstrated in Figure 1. Although the option to inject either superficial or deep to the serratus has been described, we found the superior margin of the rib that is deep to the serratus anterior muscle to be a consistent target for injection. An echogenic needle (Pajunk 18GX83, Geisingen, Germany) was advanced in-plane until the tip was positioned between the serratus anterior muscle and the rib. Twenty to forty milliliters of local anesthetic were injected into the serratus plane. Choice of local anesthetic was left to the discretion of the attending anesthesiologist on the acute pain service. Local anesthetics used for these blocks were: bupivacaine $0.25 \%$ or $0.5 \%$, with or without preservative-free dexamethasone, ropivacaine $0.2 \%$ or $0.5 \%$, or liposomal bupivacaine. In some of the single-shot nerve blocks, short-acting local anesthetics such as mepivacaine were used to hasten block onset. Liposomal bupivacaine was used alone or with bupivacaine hydrochloride $0.25 \%$, again at the discretion of the attending anesthesiologist.

Spread of the local anesthetic was visualized on ultrasound in both the cranial and caudal directions during each block. If prolonged duration of analgesia was desired, an indwelling catheter was placed using either a catheterover-needle system (Pajunk, Geisingen, Germany) or catheter-through-needle system (B Braun, Bethlehem, PA) under ultrasound guidance. After catheter placement and removal of the needle, position of the catheter was confirmed with ultrasound before the catheter was secured. Block efficacy was confirmed by performing pinprick over the rib fracture site and assessing for sensory deficit.

Indwelling catheter infusions were set at $12 \mathrm{~mL} / \mathrm{hr}$ with no bolus option of $0.2 \%$ ropivacaine using a local anesthetic infusion pump system employed by the hospital $\left(\mathrm{CADD}^{\mathrm{R}}\right.$-Solis Ambulatory Infusion Pump, Smiths Medical, St Paul, MN). There were no complications related to the procedure or the medications administered.

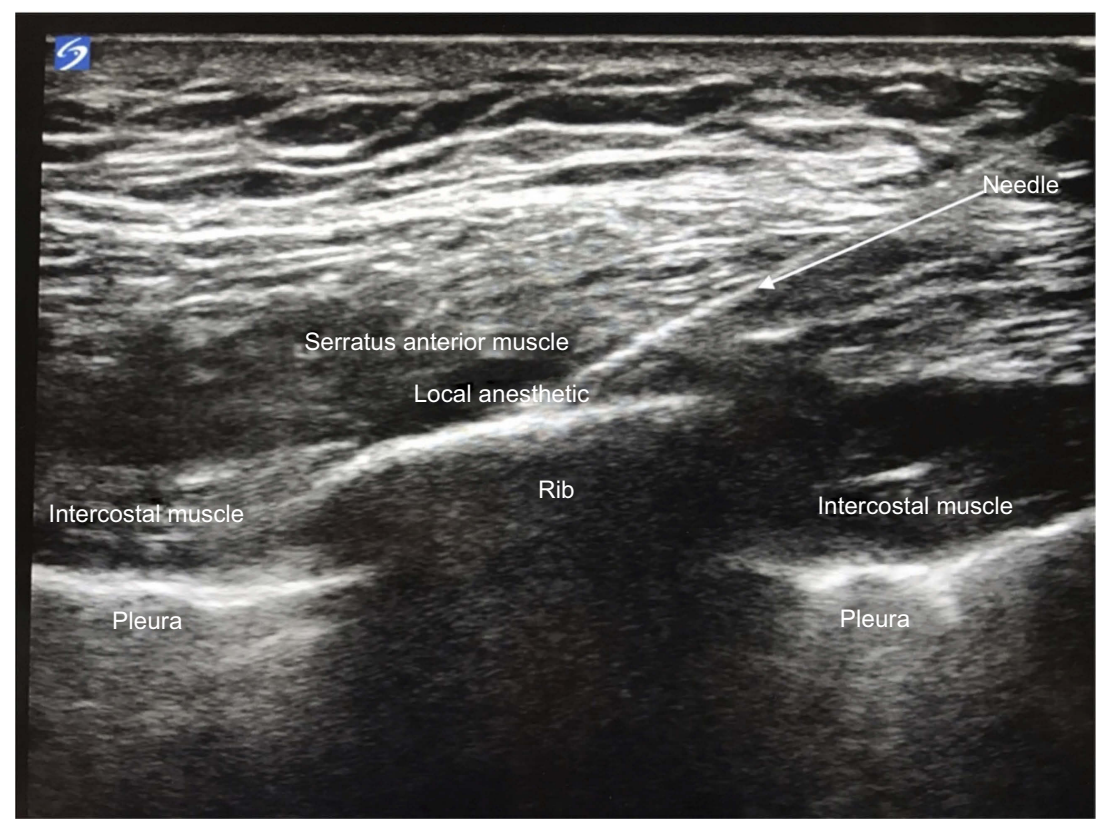

Figure I Ultrasound image of the chest wall - the ribs and pleura are identified. Tilting the ultrasound probe allows for visualization of the border between ribs, intercostal, serratus anterior, and latissimus dorsi muscles to allow for proper placement of local anesthetic and/or catheter. 


\section{Statistical analysis}

All continuous variables were summarized as median and interquartile range because they were not found to be normally distributed. Changes from baseline in pain score, $\mathrm{SpO} 2, \mathrm{RR}$, and IS were depicted by boxplot and evaluated by the Wilcoxon signed rank test. The Bonferroni correction was applied to adjust for six evaluations due to multiple endpoints as well as the measurement of IS at multiple time points. All $p$-values are two-sided and P-values less than 0.05 are considered as significant. Statistical analysis was performed using the SAS software (version 9.4, the SAS Institute, Cary, NC).

The primary outcome measured in this study was pain score on VAS. Improvements in pulse oximetry values, IS volumes, and RR were considered secondary outcomes. Based on clinical experience, it was estimated that improvement in pain score would be observed in $90 \%$ of the patients after SPB. On review of the literature, we found that a clinically significant reduction in pain score for rib fracture ranges between $30 \%$ and $50 \%$, so we aimed for at least a $30 \%$ reduction in VAS score. ${ }^{15}$

The method proposed by Noether was used to determine the approximate sample size for two-sided Wilcoxon signed rank test. ${ }^{16}$ The Bonferroni method was applied to control the family-wise type I error at $5 \%$. The size of 32 subjects is needed to provide the power of $90 \%$ for detecting significant improvement.

\section{Results}

Over the time period of August 2014 to January 2018, patients greater than 17 years of age who were admitted to the Trauma service at MHH with three or more consecutive rib fractures, and underwent ultrasound-guided SPB for pain control were included in our analysis. Thirty-four patients met criteria for inclusion in the study. Demographic data are summarized in Table 1, as medians and interquartile range, except where indicated.

Twenty-one patients were male and 13 patients were female. Median American Society of Anesthesiology Class was 3 (2-4). Median age was 57.5 years. Median number of ribs fractured was 7 . Twenty-five patients had associated pneumothorax and hemothorax.

Of the 34 patients in this study, 59\% had MRF due to MVA. Other mechanisms of injury included fall and high velocity wounds to the chest.

Baseline data are summarized in Table 2.
Table 2 Baseline clinical variables and change from baseline in pain score, incentive spirometry (IS) volume, respiratory rate, and pulse oximetry (difference between post-serratus plane block [SPB] value and baseline value).

\begin{tabular}{|c|c|c|}
\hline & $\mathbf{N}=34$ & $P$-value \\
\hline \multicolumn{3}{|c|}{ Measurements before SPB } \\
\hline Pain score & $7(6,9)$ & \\
\hline IS volume & $700(400,1000)$ & \\
\hline Respiratory rate & $24.5(18,30)$ & \\
\hline Pulse oximetry & $0.96(0.94,0.98)$ & \\
\hline \multicolumn{3}{|c|}{ Measurements after SPB } \\
\hline Pain score & $3(0,4)$ & \\
\hline IS volume at $4 \mathrm{hrs}$ & $850(590,1125)$ & \\
\hline IS volume at $24 \mathrm{hrs}$ & $775(500,1000)$ & \\
\hline IS volume at $48 \mathrm{hrs}$ & $1000(500,1100)$ & \\
\hline Respiratory rate & $16(14,19)$ & \\
\hline Pulse oximetry & $0.99(0.98,1.00)$ & \\
\hline \multicolumn{3}{|c|}{ Measurements change from baseline } \\
\hline Pain score & $-5(-7,-3)$ & $<0.001$ \\
\hline IS volume at $4 \mathrm{hrs}$ & $150(50,250)$ & $<0.001$ \\
\hline IS volume at $24 \mathrm{hrs}$ & $100(40,250)$ & $<0.001$ \\
\hline IS volume at $48 \mathrm{hrs}$ & $170(50,500)$ & 0.001 \\
\hline Respiratory rate & $-5.5(-11,-3)$ & $<0.001$ \\
\hline Pulse oximetry & $0.02(0,0.05)$ & $<0.001$ \\
\hline
\end{tabular}

Notes: Continuous values are summarized as median (IQR). Wilcoxon signed rank test was used to evaluate change from baseline in pain score, IS volume, respiratory rate, and pulse oximetry. Bonferroni adjusted $P$-values were calculated to control the overall type I error of six tests

Pain scores were extracted from the electronic medical record in a retrospective fashion. The NRS was used, ranging from 0 to 10 with 0 being no pain and 10 being the worst possible pain. All assessments were made by the bedside nurse. Time of SPB placement was recorded by the acute pain service. Pain scores retrieved from the chart were from the immediate post-block period that reflects the onset of local anesthetic (0.5-4 hrs) or when nursing assessments were done after block was performed (0.5-8 hrs). Pain scores decreased from a median of 7 to 3 after block onset. No further pain scores were reviewed, as the duration of analgesia varied depending on whether the patient received a single-shot block or an indwelling catheter. According to these data (see Figure 2), SPB was effective in reducing pain scores $0.5-4 \mathrm{hrs}$ after injection of local anesthetic.

We systematically examined the effect of SPB on ventilatory function. IS volumes were retrieved retrospectively. All assessments were made by the acute pain 


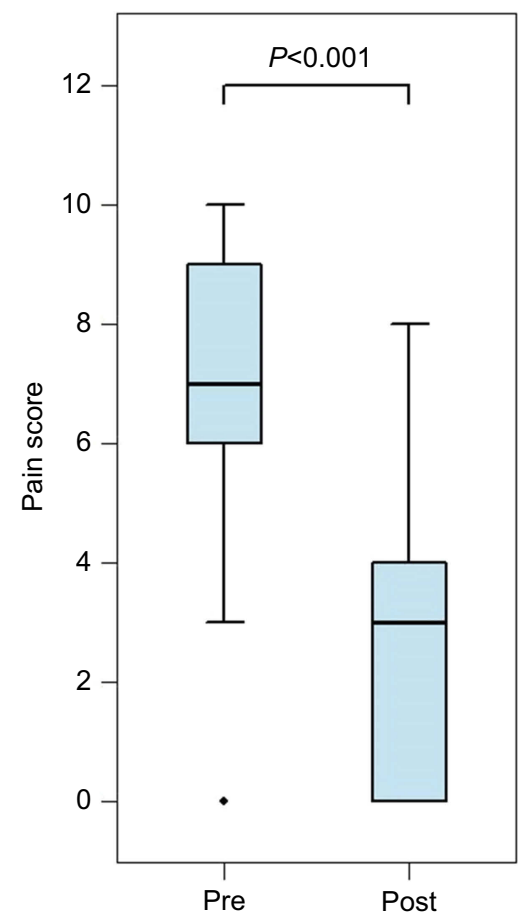

Figure 2 Pain scores-pain scores before and after SPB are depicted by boxplot. Changes in pain score $(\mathrm{N}=33)$ were analyzed by the Wilcoxon signed rank test. Bonferroni adjusted correction was applied to control the overall type I error of six comparisons. $P$-value is $<0.001$.

Abbreviation: SPB, serratus plane block.

nurse or the respiratory therapist. IS volumes that were retrieved were within $0.5-4 \mathrm{hrs}$ after the block was placed, signifying the onset of the different local anesthetics used, ranging from 0.5 to $8 \mathrm{hrs}$, at $8-24$ and $24-48 \mathrm{hrs}$. IS volumes were measured with the Airlife volumetric spirometer. IS volumes at 4,24 , and 48 hrs improved significantly by median changes of 150,175 , and $300 \mathrm{~mL}$, respectively, after the SPB block (see Figure 3). IS volumes are effort dependent and improve with active patient coaching. IS volumes obtained $4 \mathrm{hrs}$ after the block were measured by members of our team, and volumes at 24 and $48 \mathrm{hrs}$ were obtained by respiratory therapists. This may explain the decrease in change from baseline seen at $24 \mathrm{hrs}$ as opposed to at $4 \mathrm{hrs}$ after the block.

Next, we turned our attention to the effect of SPB on RR. All assessments were made by the bedside nurse or the respiratory therapist. RR retrieved from the chart was from the immediate post-block period that reflects the onset of local anesthetic (0.5-4 hrs) or when assessments were done after the block was performed, ranging from 0.5 to $24 \mathrm{hrs}$. RR was measured with respiratory impedance

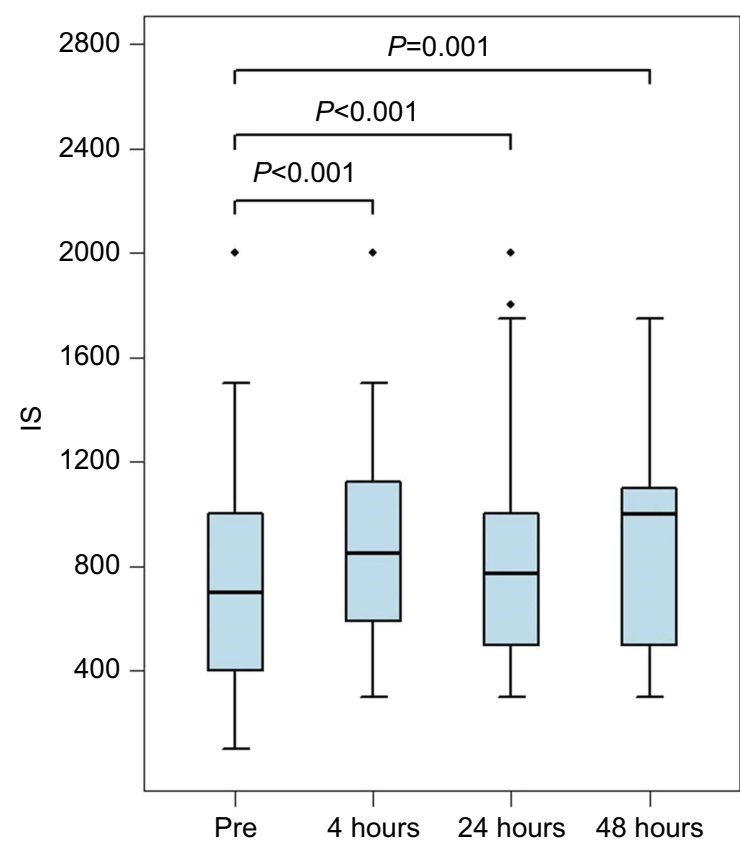

Figure 3 IS before and $4 \mathrm{hrs}, 24 \mathrm{hrs}, 48 \mathrm{hrs}$ after SPB is depicted by boxplot. Changes in IS from baseline ( $N=28,25,21$ for 4,24 , and $48 \mathrm{hrs}$, respectively) were analyzed by the Wilcoxon signed rank test. Bonferroni correction was applied to control the overall type I error of six comparisons. $P$-values are $<0.00 I$ at 4 and 24 hrs. $P$-value at $48 \mathrm{hrs}$ is $0.00 \mathrm{I}$.

Abbreviations: IS, incentive spirometry; SPB: serratus plane block.

monitoring. Median values for RR decreased significantly from 24.5 to 16 breaths per minute (see Figure 4).

Pulse oximetry ( $\mathrm{SpO} 2)$ values were reviewed. All assessments were made by the bedside nurse; data retrieved from the chart were from the immediate post-block period that reflects the onset of local anesthetic (0.5-4 hrs) or when assessments were done after the block was performed, ranging from 0.5 to $24 \mathrm{hrs}$. There was a statistically significant improvement in $\mathrm{SpO} 2$ values $(\mathrm{P}<0.001)$. SpO2 improved from a median value of $96-99 \%$ within $24 \mathrm{hrs}$ after SPB (see Figure 5). However, the clinical significance is more dramatic when the required degree of oxygen supplementation is considered: most patients had considerable decreases in required $\mathrm{FiO} 2$. Of the 34 patients in the study, 32 experienced either an improvement in their pulse oximetry value or their supplemental oxygen requirement. Some patients demonstrated this by ability to wean off of high flow nasal oxygen; others were weaned completely from supplemental oxygen to room air. Two patients were able to be extubated, and SPB prevented impending intubation in one patient. In another, scheduled surgical fixation of the fractured ribs was canceled due to improved respiratory mechanics following SPB. 


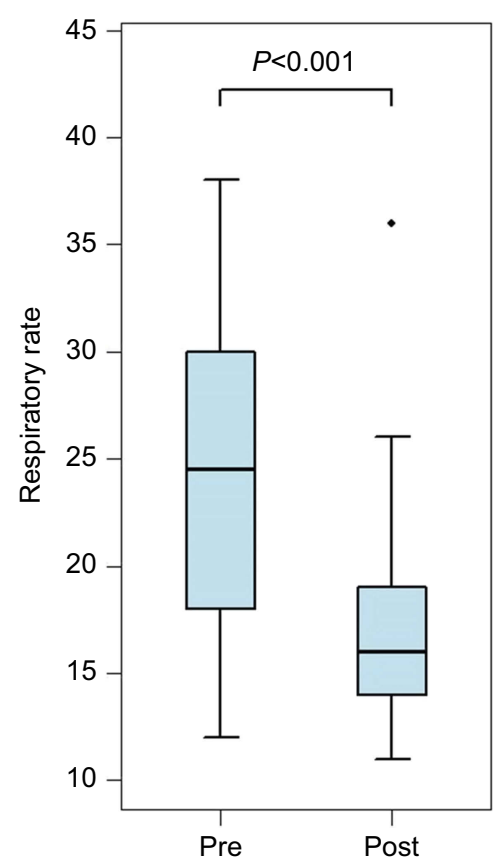

Figure 4 Respiratory rates before and after SPB are depicted by boxplot. Changes in respiratory rate $(\mathrm{N}=34)$ were analyzed by the Wilcoxon signed rank test. Bonferroni correction was applied to control the overall type I error of six comparisons. $P$-value is $<0.001$.

Abbreviation: SPB, serratus plane block.

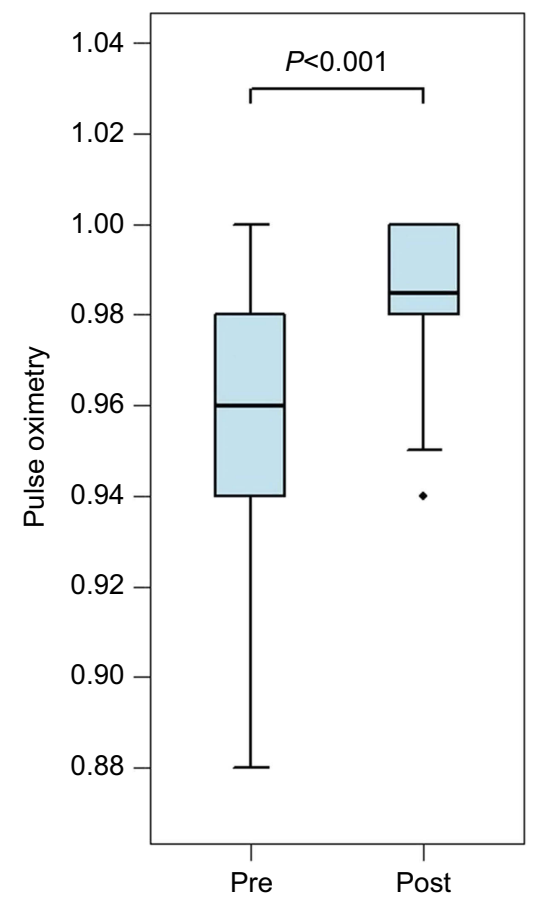

Figure $5 \mathrm{SpO} 2$ before and after SBP is depicted by boxplot. Changes in $\mathrm{SpO} 2$ $(\mathrm{N}=34)$ were analyzed by the Wilcoxon signed rank test. Bonferroni correction was applied to control the overall type I error of six comparisons. $P$-value is $<0.001$. Abbreviation: SPB, serratus plane block.

\section{Discussion}

SPB is a novel technique for delivering regional anesthesia to the hemithorax. In this cohort of trauma patients with MRF, the authors found that SPB improved pain scores and IS volumes. To the authors' knowledge, this is the first study where data (pain score, IS volumes, RR, SpO2) were reviewed systematically in a retrospective fashion before and after the SPB.

There are a variety of multimodal analgesic regimens, regional, and neuraxial techniques that can be considered to minimize respiratory compilations and reduce morbidity in patients with MRF. ${ }^{5}$ TEA and thoracic paravertebral block (TPVB) are currently accepted to be the gold standards and have shown similar efficacy to each other. ${ }^{17}$ Despite clear superiority in pain management, neuraxial analgesia has demonstrated no significant benefit on mortality or intensive care unit or hospital lengths of stay. ${ }^{18}$ This may be due to the propensity of TEA to cause sympathetic blockade, which results in vasodilation, venous pooling, and a subsequent decrease in venous return. ${ }^{19}$ TEA can also inadvertently block the cardiac accelerator fibers, which are at thoracic levels 1 through 4, making it difficult for patients to compensate hemodynamically for the hypotension. Anecdotally, TPVB generally results in less hypotension because it is a unilateral sympathectomy when compared to TEA, but it carries a similar risk of epidural hematoma in patients receiving chemical VTE prophylaxis. TEA and TPVB are also technically challenging and carry a risk of spinal cord injury, spinal epidural hematoma, and refractory hypotension. Positioning for TEA and TPVB can also be challenging in trauma patients with multiple extremity fractures, pelvic fractures, or unstable fractures of the spinal column.

SPB has been shown to maintain hemodynamic stability and provide comparable analgesic effects to thoracic epidural for thoracotomy pain. ${ }^{20}$ Therefore, SPB may be used in trauma patients with contraindications to neuraxial techniques. SPB targets the lateral cutaneous branch of the thoracic intercostal nerves providing analgesia to the anterior-lateral chest wall extending from mid-clavicular line to mid-scapula. Chemical VTE prophylaxis does not need to be interrupted for placement of SPB, as this procedure is not subjected to the same anticoagulant guidelines. SPB has been a frequently studied regional anesthetic technique, gaining popularity over the last 5 years. Serious complications have not yet been reported with this technique. 
The craniocaudal extent of the analgesia is not fully understood. The original description of SPB is a modification of the Pecs Type II block in which analgesia was achieved consistently from $\mathrm{T} 2$ to $\mathrm{T} 4$ dermatomes and variable spread to $\mathrm{T} 6$ using an injection point at the anterior axillary line between pectoralis minor muscle and the serratus anterior muscle. ${ }^{21}$ For SPB, Blanco et al, demonstrated anesthesia from $\mathrm{T} 2$ to $\mathrm{T} 9$ in all four volunteers when using $0.4 \mathrm{~mL} / \mathrm{kg}$ of local anesthetic ( $28 \mathrm{~mL}$ for a $70 \mathrm{~kg}$ patient) injected either superficial or deep to the serratus anterior muscle at the midaxillary line. ${ }^{10}$ A recent study of injection volumes in SPB in 42 patients showed that $40 \mathrm{~mL}$ of $0.375 \%$ ropivacaine anesthetized on average six dermatomes compared to four dermatomes with $20 \mathrm{~mL}$ of $0.375 \%$ ropivacaine without any effect on duration of analgesia. ${ }^{22}$ In a cadaveric study, $40 \mathrm{~mL}$ of agitated saline was injected into the posterior axillary line followed by sonographic confirmation of injectate spread from the 2nd intercostal space to subcostal margin. ${ }^{23}$ Despite reports that the extent of analgesia is limited to the lateral cutaneous branch of intercostal nerves $\mathrm{T} 2-\mathrm{T} 9$, in this study SPB with $30 \mathrm{~mL}$ of local anesthetic relieved rib fracture pain in one patient with 1st and 2nd rib fractures and in another patient with 9th-12th rib fractures. This may suggest the osteotomal coverage is wider than cutaneous coverage. We also found that injection point (anterior axillary line vs mid-axillary line from the 3rd to the 7th rib) did not affect extent of analgesia in our patients.

This study is limited by its retrospective nature, small size, and single-institution experience. Total opioid consumption and length of stay in the hospital were not included as these patients who had MRF had concomitant distracting injuries. Recorded pain scores may also be a limitation, as pain scores recorded by the bedside nurse may be reflective of other injuries and not solely rib fracture pain. Further, because formal spirometry is not routinely performed at our institution, IS volumes were used as a surrogate measurement of vital capacity. Although IS depends upon patient effort, a study of pulmonary lobectomy patients showed IS to be a reliable indicator of pulmonary function and correlates well with vital capacity. ${ }^{24}$ Further studies comparing neuraxial techniques to SPB are needed to assess their relative efficacy.

\section{Conclusions}

SPB is a minimally invasive, superficial, regional analgesic technique. Our study concluded that SPB decreases NRS pain scores and RR; it also demonstrated an increase in both IS volumes and $\mathrm{SpO} 2$ values. In polytrauma patients, ultrasound-guided SPB is advantageous over neuraxial techniques for MRF because it is not limited by patient positioning, does not cause hypotension, and VTE chemoprophylaxis does not need to be interrupted. Although this retrospective study demonstrates the positive impact of the SPB on NRS, IS, SpO2 values, and RR, further prospective research is needed to show analgesic efficacy of SPB vs TEA and TPVB.

\section{Acknowledgments}

The authors would like to acknowledge Renuka George, $\mathrm{MD}$, and $\mathrm{Xu}$ Zhang, $\mathrm{PhD}$, for their assistance in the completion of this work. The authors have no sources of funding to declare for this manuscript.

\section{Author contributions}

All authors contributed towards data analysis, drafting and critically revising the paper, gave final approval of the version to be published, and agreed to be accountable for all aspects of the work.

\section{Disclosure}

Dr Holger Eltzschig reports grants from the United States NIH, and an honorarium from Novartis Pharma AG, outside the submitted work; and reports no other conflicts of interest in this work. The other authors declare no conflicts of interest in this work.

\section{References}

1. Murphy CET, Raja AS, Baumann BM, et al. Rib fracture diagnosis in the Panscan Era. Ann Emerg Med. 2017;70(6):904-909. doi:10.1016/j. annemergmed.2017.04.011

2. Pitts SR, Niska RW, Xu J, Burt CW. National Hospital Ambulatory Medical Care Survey: 2006 emergency department summary. Natl Health Stat Report. 2008;7:1-38.

3. Haenel JB, Moore FA, Moore EE, Sauaia A, Read RA, Burch JM. Extrapleural bupivacaine for amelioration of multiple rib fracture pain. J Trauma. 1995;38(1):22-27.

4. Fusco P, Scimia P, Di Carlo S, et al. A case report on ultrasoundguided serratus plane block and fast-track tracheal extubation in the operating room for thoracic trauma patients: a case report. A A Case Rep. 2017;9(11)305-307. doi: 10.1213/XAA.0000000000000600

5. Okmen K, Okmen BM. The efficacy of serratus anterior plane block in analgesia for thoracotomy: a retrospective study. J Anesth. 2017;31 (4):579-585. doi:10.1007/s00540-017-2364-9

6. Sabanathan S, Mearns AJ, Bickford Smith PJ, et al. Efficacy of continuous extrapleural intercostal nerve block on post-thoracotomy pain and pulmonary mechanics. Br J Surg. 1990;77(2):221-225.

7. Mackersie RC, Karagianes TG, Hoyt DB, Davis JW. Prospective evaluation of epidural and intravenous administration of fentanyl for pain control and restoration of ventilatory function following multiple rib fractures. J Trauma. 1991;31(4):443-449; discussion 449-451. 
8. Horlocker TT, Wedel DJ, Rowlingson JC, et al. Regional anesthesia in the patient receiving antithrombotic or thrombolytic therapy: American Society of Regional Anesthesia and Pain Medicine Evidence-Based Guidelines (Third edition). Reg Anesth Pain Med. 2010;35(1):64-101.

9. Moore DC, Bridenbaugh LD. Intercostal nerve block in 4333 patients: indications, technique, and complications. Anesth Analg. 1962;41:1-11.

10. Blanco R, Parras T, McDonnell JG, Prats-Galino A. Serratus plane block: a novel ultrasound-guided thoracic wall nerve block. Anaesthesia. 2013;68(11):1107-1113. doi:10.1111/anae.12344

11. Durant E, Dixon B, Luftig J, Mantuani D, Herring A. Ultrasoundguided serratus plane block for ED rib fracture pain control. Am J Emerg Med. 2017;35(1):197e193-197e196. doi:10.1016/j. ajem.2016.07.021

12. Lopez-Matamala B, Fajardo M, Estebanez-Montiel B, Blancas R, Alfaro P, Chana M. A new thoracic interfascial plane block as anesthesia for difficult weaning due to ribcage pain in critically ill patients. Med Intensiva. 2014;38(7):463-465. doi:10.1016/j. medin.2013.10.005

13. Kunhabdulla NP, Agarwal A, Gaur A, Gautam SK, Gupta R, Agarwal A. Serratus anterior plane block for multiple rib fractures. Pain Physician. 2014;17(5):E651-E653.

14. Bossolasco M, Bernardi E, Fenoglio LM. Continuous serratus plane block in a patient with multiple rib fractures. J Clin Anesth. 2017;38:85-86. doi:10.1016/j.jclinane.2016.12.015

15. Peek J, Smeeing DPJ, Hietbrink F, Houwert RM, Marsman M, de Jong MB. Comparison of analgesic interventions for traumatic rib fractures: a systematic review and meta-analysis. Eur J Trauma Emerg Surg. 2018. doi:10.1007/s00068-018-0918-7

16. Noether GE. Sample size determination for some common nonparametric tests. J Am Stat Assoc. 1987;82(398):645-647. doi:10.1080/ 01621459.1987 .10478478
17. Mohta M, Verma P, Saxena AK, Sethi AK, Tyagi A, Girotra G. Prospective, randomized comparison of continuous thoracic epidural and thoracic paravertebral infusion in patients with unilateral multiple fractured ribs-a pilot study. J Trauma. 2009;66(4):1096-1101. doi:10.1097/TA.0b013e318166d76d

18. Carrier FM, Turgeon AF, Nicole PC, et al. Effect of epidural analgesia in patients with traumatic rib fractures: a systematic review and meta-analysis of randomized controlled trials. Can J Anaesth. 2009;56(3):230-242. doi:10.1007/s12630-009-9052-7

19. Baidya DK, Khanna P, Maitra S. Analgesic efficacy and safety of thoracic paravertebral and epidural analgesia for thoracic surgery: a systematic review and meta-analysis. Interact Cardiovasc Thorac Surg. 2014;18(5):626-635. doi:10.1093/icvts/ivt551

20. Khalil AE, Abdallah NM, Bashandy GM, Kaddah TA-H. Ultrasoundguided serratus anterior plane block versus thoracic epidural analgesia for thoracotomy pain. J Cardiothorac Vasc Anesth. 2017;31 (1):152-158. doi:10.1053/j.jvca.2016.08.023

21. Blanco R, Fajardo M, Parras Maldonado T. Ultrasound description of Pecs II (modified Pecs I): a novel approach to breast surgery. Rev Esp Anestesiol Reanim. 2012;59(9):470-475. doi:10.1016/j. redar.2012.07.003

22. Kunigo T, Murouchi T, Yamamoto S, Yamakage M. Injection volume and anesthetic effect in serratus plane block. Reg Anesth Pain Med. 2017;42(6):737-740. doi:10.1097/AAP.0000000000000649

23. Daga V, Narayanan MK, Dedhia JD, Gaur P, Crick H, Gaur A. Cadaveric feasibility study on the use of ultrasound contrast to assess spread of injectate in the serratus anterior muscle plane. Saudi J Anaesth. 2016;10(2):198-201. doi:10.4103/1658-354X.168825

24. Bastin R, Moraine JJ, Bardocsky G, Kahn RJ, Melot C. Incentive spirometry performance. A reliable indicator of pulmonary function in the early postoperative period after lobectomy? Chest. 1997;111 (3):559-563. doi:10.1378/chest.111.3.559

\section{Publish your work in this journal}

Local and Regional Anesthesia is an international, peer-reviewed, open access journal publishing on the development, pharmacology, delivery and targeting and clinical use of local and regional anesthetics and analgesics. The journal welcomes submitted papers covering original research, basic science, clinical studies, reviews \& evaluations, guidelines, expert opinion and commentary, case reports and extended reports. The manuscript management system is completely online and includes a very quick and fair peer-review system, which is all easy to use. Visit http://www.dovepress.com/testimonials. php to read real quotes from published authors. 